\title{
À propos de A Papuan Plutocracy. Ranked Exchange on Rossel Island de John LIEP
}

\section{Gilles Bounoure}

\section{(2) OpenEdition \\ Journals}

Édition électronique

URL : http://journals.openedition.org/jso/6357

DOI : $10.4000 /$ jso.6357

ISSN : $1760-7256$

Éditeur

Société des océanistes

Édition imprimée

Date de publication : 30 juin 2011

Pagination : 183-188

ISBN : 978-2-85430-030-7

ISSN : 0300-953x

Référence électronique

Gilles Bounoure, «À propos de A Papuan Plutocracy. Ranked Exchange on Rossel Island de John LIEP », Journal de la Société des Océanistes [En ligne], 132 | 1er semestre 2011, mis en ligne le 30 juin 2011, consulté le 24 septembre 2020. URL : http://journals.openedition.org/jso/6357 ; DOI : https://doi.org/ $10.4000 / j s 0.6357$ 


\title{
MISCELLANÉES
}

\section{À propos de A Papuan Plutocracy. Ranked Exchange on Rossel Island de John LIEP ${ }^{1}$}

par

\author{
Gilles BOUNOURE*
}

Fruit de plus de trente-cing ans de préparation et de réflexion, ce livre à la présentation soignée impressionne par son ampleur et l'importance des sujets qu'il aborde. Signaler sa publication est indispensable, même s'il est impossible ici de passer en revue et a fortiori de discuter toutes les questions anciennes qu'il rouvre et les nouvelles qu'il pose ou suggère, à propos d'un " cas d'école ". Dans un rapport de 1893 (reproduit dans Armstrong 1978 [1928], pp. 209-213), le commissaire spécial pour la Nouvelle-Guinée britannique et ethnographe William McGregor semble être le premier à avoir souligné l'intérêt pour l'anthropologie de l'île Rossel ou Yela, isolée à l'extrémité orientale de l'archipel de la Louisiade, à $375 \mathrm{~km}$ à vol d'oiseau de la pointe est de la Nouvelle-Guinée, isolement expliquant à ses yeux que ses habitants soient non seulement les seuls de langue papoue dans l'archipel et même la région, mais " peut-être les Papous de sang le plus pur de toute la Possession ». Dans le même esprit, la première monographie ethnologique qui leur fut consacrée, due à Armstrong, s'ouvrait sur une introduction mi-médicale mi-diffusionniste d'Alfred Cort Haddon, à laquelle répondait en appendice une série de relevés anthropométriques, principalement craniométriques, tendant à mettre en évidence les différences physiques des insulaires par rapport à leurs plus proches voisins.
Surtout, précédée en 1924 dans The Economic Journal par un article annonçant la découverte d'un " système monétaire unique " propre à Rossel, cette monographie lui consacrait deux chapitres ("The Monetary System ", "Monetary Ceremonial ») qui allaient inspirer de nombreux commentateurs, notamment Paul Einzig (1949 : chapitre " The intricate currency of Rossel Island ", pp. 72-75), partagé entre admiration (" one of the most complicated systems of means of payment ever devised by tortuous human mind ", " this extraordinary system ", etc.) et doute (" a monetary system - if it can be regarded as such ", " the monetary status of ndap and nko is open to doubt ", etc.). Des travaux récents (par exemple Goldberg, 2005) continuent à en tirer argument pour contester l'ancienneté, et plus encore les origines "primitives ", de la monnaie fiduciaire, ou fiat money des Anglo-Saxons. Si, comme l'assurent de nombreux observateurs, la crise financière actuelle paraît sonner le glas de la "Fiat Money Era ", elle pourrait susciter encore nombre de réflexions s'appuyant sur le « système monétaire unique " de Rossel, quelques réserves qu'appellent les rapprochements entre les " paléo-monnaies " d'Océanie ou d'ailleurs et les moyens d'échange et de paiement de l'économie marchande et financière d'aujourd'hui.

Dans son introduction, J. Liep décrit avec une élégance et une franchise rares le paysage intel-

2009. Aarhus, Aarhus University Press, XxxvıII-376 p., glossaire, bibliographie, index, cartes, schémas, nombreuses ill. dans le texte.

flongue@wanadoo.fr 
lectuel du milieu des années 1960 qui l'amena à se saisir de ces questions jusqu'à devenir non seulement le spécialiste incontesté de Rossel et de son "système ", mais aussi une autorité dans maints domaines connexes, la région Massim pour commencer, et plus largement les sociétés mélanésiennes. La direction de ses travaux et leur orientation critique doivent beaucoup à un article de George Dalton (1965) qui reprochait à Malinowski et à Firth de tirer leur conception de la "vraie monnaie " des attributs monétaires observables dans l'économie occidentale de marché (" crucial misunderstanding ") et qui développait, à titre de contreexemple, une longue analyse du " système monétaire " de Rossel d'après Armstrong. Il y pointait les "fautes" de cet " économiste qui avait joué à l'anthropologue " et n'avait ainsi apporté qu'une seule leçon, non sur la nature de la monnaie primitive, mais sur les erreurs à éviter en anthropologie. Une longue note sur les "problèmes non résolus " comportait une série de questions à éclaircir pour " comprendre pleinement le système » de Rossel, définissant un véritable programme de recherche. Le triomphe de J. Liep est non seulement de l'avoir rempli, mais d'être allé très au-delà, notamment grâce au principe de ce qu'il nomme "l'analyse dynamique ", attentive aux changements historiques.

Ce principe se retrouve dans le plan même de l'ouvrage, qui s'ouvre sur un "prologue » dévolu aux "Massim Transformations ", où l'auteur déploie un talent de la synthèse et une clarté d'exposition tout à fait remarquables. À cette leçon d'histoire qui devrait à elle seule attirer vers ce livre à la fois l'ensemble des océanistes et tous ceux qui s'intéressent au cycle de la kula, succèdent deux parties, "The Setting ", quatre chapitres qu'on pourrait dire d'excellente ethnologie " classique ", et " Ranked Exchange ", cinq chapitres consacrés non pas au "système monétaire " au sens d'Armstrong, mais plutôt au système social qui en est la clé, dans les divers sens du terme. L'épilogue vient justifier le qualificatif de "ploutocratie papoue " choisi par l'auteur pour décrire cette société qu'il a passé tant de temps à côtoyer, scruter et étudier. Le résultat est-il convaincant ? À première lecture, l'ouvrage emporte l'adhésion, mais dès sa parution des critiques se sont fait jour, notamment sous la plume malicieuse de Joel Robbins (2009), reprochant à J. Liep de brosser un tableau à la fois trop " satisfaisant " et trop peu cohérent de la société rosselienne, égalitaire et inégalitaire à la fois. Faute de connaître en détail la réponse que J. Liep entendait lui apporter au colloque ESfO de 2010 ("Conflicting Values : Reciprocity and Self-Enhancement in Melanesia »), on se limitera ici à signaler l'existence de ce premier débat qui témoigne au moins de l'importance de ce livre, et à livrer quelques remarques suggérées par sa lecture.

Il convient d'abord de noter qu'à la différence d'Armstrong et de ses descriptions simplistes, J. Liep dresse un tableau nuancé - et par conséquent largement plus complexe encore - du système des échanges en vigueur à Rossel. Il en prévient d'avance (p. 167), la frontière est souvent floue entre les « monnaies " de coquillage (ndap et $k \hat{e}$, en spondyle et Chama) et les « objets de valeur » susceptibles eux-mêmes d'être échangés, telles les lames de haches cérémonielles en diorite ou rhiolithe et les spatules à chaux généralement importées, ou les colliers de coquillages exportés depuis Rossel dans l'ensemble de l'aire Massim et auxquels il est le premier à consacrer une étude détaillée (pp. 203-208). De son examen de leurs modes récents de circulation, il dégage un tableau qui n'est pas "nettement tranché ", et sans " explication complète de leurs séparations et combinaisons variées" (p. 209) : les ndap sont de rigueur pour les compensations matrimoniales, les fêtes à cochons, les paiements concernant les maisons ou les embarcations et les échanges funéraires, les kê n'entrent en jeu que dans les trois premiers cas de figure, les autres objets de valeur dans les deux derniers, mais un peu aussi lors des fêtes, tandis que le papier-monnaie, qui a sa pleinement sa place dans les fêtes, semble aussi s'insinuer jusque dans les échanges funéraires.

Son analyse des "procédures financières " (pp. 298-319) conduit l'auteur à distinguer six types usuels de circulation des ndap et des $k \hat{e}$, le dépôt de garantie et la caution, restitués une fois effectuée une opération de moindre valeur, le remplacement d'une pièce de grande valeur par d'autres totalisant une valeur équivalente, le cadeau pour réclamer un paiement de plus haut prix, la mise en gage pour obtenir un prêt ou une prestation de valeur supérieure, et la substitution compensatoire, pour accompagner le remplacement d'un ndap de grand prix par un lot de moindre valeur. Mais s'agissant de « monnaies " qui n'ont rien d'anonyme, c'est à ceux qui connaissent leur histoire, les anciens, et parmi eux spécialement ceux qui sont ou ont été dépositaires ou propriétaires de ces trésors, d'établir leur cours dans chacun des échanges, ou plutôt des négociations, où ils interviennent. Si les jeunes gens, ajoute J. Liep, se plaignent de l'âpreté des anciens dont ils attendent la disparition pour abolir ces échanges tellement inégaux, les pratiques rosseliennes, insiste-t-il aussi, sont en contradiction évidente avec la théorie du don et du contre-don, et tout ce qu'elles supposent de générosité et de solidarité dans les sociétés océaniennes.

Sur le plan de la méthode, l'« analyse dynamique " dont se prévaut l'auteur consiste aussi à en finir avec les tromperies du " présent ethnologique " (d'où beaucoup d'hypothèses his- 
toriques extrêmement vraisemblables dans ce livre, mais qu'on lui contestera sans doute) et avec nombre d'autres fausses certitudes générées par l'esprit de système propre aux Occidentaux. Parmi celles-ci, il est très réjouissant de lire tout ce que J. Liep a pu relever d'influences austronésiennes venues de la région Massim dans cette société papoue de Rossel qu'Armstrong estimait au contraire remarquable par son «isolement ». C'est en particulier la numération décimale d'origine austronésienne qui s'applique au classement moins arithmétique ou financier que hiérarchique des «monnaies ". Dans le contexte clairement austronésien de Malaita, Daniel De Coppet (1970) avait souligné l'importance dans la hiérarchie monétaire des 'Are'Are d'une symbolique des nombres se fixant principalement sur le "sept hiu» et le "neuf siwa", et dont la survivance s'observe quotidiennement dans les sociétés occidentales. Pour Rossel, l'ouvrage d'Armstrong (1978 [1928], p. 148 et p. 151), qui n'est pas totalement à oublier du point de vue ethnographique, livre quelques remarques significatives sur les restrictions entourant le sept et le neuf, obligeant par exemple les pêcheurs venus à Loa, îlot à l'est de l'île principale, à ne rapporter de poissons que par lots de dix dans chaque canot, sauf à devoir rejeter leurs prises à la mer, le nombre neuf devant surtout être évité. Cette importance accordée à la dizaine se retrouve les paiements, cordes de dix fragments de Chama pour la "monnaie féminine " $k \hat{e}$, alignements par dix de la " monnaie masculine " ndap en coquille polie de spondyle. J. Liep n'y a pas prêté attention, et il $\mathrm{y}$ a peut-être là matière à prolonger cette recherche pourtant particulièrement volumineuse et riche. Jusqu'où pousser les analyses et les comparaisons, c'est un autre sujet important de ce livre.

Les lecteurs informés seront sensibles au souci de l'auteur de limiter rigoureusement ses références à ce qu'il appelle des parallèles pertinents, le plus souvent limités à la région Massim. Pour cette dernière, il prend nettement ses distances à l'égard de l'ouvrage bien connu d'Annette Wiener (1992) auquel il objecte d'abuser du terme "inaliénable ", les biens les plus précieux de la kula ne l'étant jamais tout à fait, de réifier les "possessions " au détriment des conditions sociales qui les fondent, et d'écarter comme des "paradoxes " les cas de cession de ces objets, ce qui l'amène à cette conclusion :

«Elle a ainsi utilisé un terme absolu pour analyser une large gamme de limitations hiérarchiques à l'aliénation de la propriété. Il est plus adapté de considérer l'inaliénabilité comme le point final d'un continuum dont l'autre point est l'aliénabilité, et de parler d'aliénabilité restreinte pour toutes les situations intermédiaires. » (Liep, 2009 : 11)
Avec la même prudence, s'il fait allusion (comme Dalton déjà) aux étranges "monnaies » micronésiennes, c'est surtout du fait qu'elles échappent spectaculairement au système divisionnaire usité depuis longtemps en Occident. Évoquant l'ancienne institution de la prostitution à Rossel ( " communal mistress ", écrit Armstrong), il se réfere à ce qu'a rapporté Roheim de Normandby, mais ce qu'il en écrit lui-même suggère de très nombreux parallèles, $\mathrm{y}$ compris du point de vue " monétaire », avec l'archipel de l'Amirauté, celui des Salomon ou même Santa Cruz, dont la célèbre "monnaie de plumes" a des liens bien connus avec les "Amüsierdamen " (comme disait Felix Speiser), relation observée également dans plusieurs civilisations de Micronésie. Voilà un point parmi d'autres où la lecture de cet ouvrage est excitante et propre à susciter de nouvelles recherches.

Une autre question décisive porte sur le sens $\mathrm{du}$ caractère inaliénable de certains biens de prestige (ndap) des insulaires de Rossel, placés plus ou moins absurdement par Armstrong au sommet de son système monétaire, comme s'ils servaient de "garantie bancaire " aux moyens d'échange proprement dits (J. Liep parle lui-même de « security "). Là encore, d'un point de vue " monétariste ", les parallèles ne manqueraient pas dans les sociétés mélanésiennes, entre les " têtes de monnaie " kanak, les charmes associés aux "monnaies " de Santa Cruz ou les kia de Mono-Alu (Monnerie, 2002) et les objets analogues des Tolai (a vaimkor, charme empêchant de tout dépenser du tambu selon Epstein, 1979 : 166) ou d'autres biens inaliénables et inconvertibles ayant existé en Nouvelle-Irlande centrale (Derlon, 2002 : 160) et septentrionale (Küchler, 2002 : 174-175). Comme tend à le montrer J. Liep, il s'agit certainement là de vraies "valeurs " pourvu de donner à ce terme un sens non pas financier, mais sociologique sinon moral ou " religieux ». Mauss (1969 [1914]) parlait déjà du " caractère religieux et magique de la monnaie " primitive, "notion " qu'il estimait associée à « celle de pouvoir magique ». Il ajoutait :

«Le pouvoir d'achat de la monnaie primitive c'est avant tout, selon nous, le prestige que le talisman confere à celui qui le possède et qui s'en sert pour commander aux autres. »

Certainement, mais à quelles fins ? Selon J. Liep, "les règles et pratiques des échanges hiérarchisés » (titre de son chapitre 10) tendaient en fin de compte à préserver l'auto-reproduction de cette société au profit des détenteurs de " monnaie », bien au-delà du " protectionnisme » économique ou de l'endogamie, avec ce souci de la différenciation culturelle que semblent avoir partagé tant de populations mélanésiennes, y compris les plus commerçantes. Mais le rôle du ndaapi ou «money man » que l'ouvrage n'évoque 
qu'une fois sous ce nom (p. 82, ndeb chez Armstrong, p. 66), et maintes fois sous l'appellation de "big man ", n'est-il pas comparable à celui $\mathrm{du}$ " trésorier de lignage " décrit par Michel $\mathrm{Pa}$ noff chez les Maenge, qu'il présente comme une société "à peu près égalitaire " et non comme une oligarchie financière (1980:16-17 et 19) ? Quant à la «transformation " des précieux ndap en biens inaliénables, inimitables et non remplaçables, résulte-t-elle réellement d'une destruction accidentelle dont J. Liep pense avoir reconstitué l'histoire (pp. 189-191), ou au-delà de cette explication évhémériste faut-il y voir l'effet d'une politique plus ancienne de numerus clausus clairement conservatrice, sans préjudice des gestes de destruction ou d'enfouissement des biens inaliénables largement attestés ailleurs, notamment en Nouvelle-Bretagne et aux Salomon ?

Contrôle des échanges et maintien des hiérarchies par la thésaurisation, voilà en somme en quoi consisterait cette " ploutocratie papoue". Ce titre semble paradoxal à propos d'une île au "sol apparemment très pauvre », peuplée par des " gens pauvres et sauvages " (Le Hunte, 1899, in Armstrong, 1978 [1928] : 214), et dont l'auteur et son préfacier Stephen C. Levinson indiquent tous deux que faute d'intérêts miniers comme à Misima, autre île de la Louisiade, elle est totalement délaissée par les autorités, y compris à propos du SIDA qui pourrait la menacer gravement. Non sans un certain sens du suspens, J. Liep ne justifie son titre que dans les deux dernières pages de son livre, et principalement d'après un précédent anthropologique, l'intitulé donné en 1922 par Malinowski à l'une des sections de ses Argonautes, "Chefferie : pouvoir par la richesse; une communauté ploutocratique ", mais sans utiliser le terme dans le texte, comme l'observe aussi J. Liep. Trait d'époque ou influence de Malinowski, Roheim parlait l'année suivante, à propos de la Nouvelle-Bretagne et des Tolai qu'il ne nomme pas, "d'une forme assez évoluée de la société capitaliste : intérêts élevés, combinaisons illicites, arrogance ploutocratique et aussi escroquerie en tout genre " (1978 [1923] : 217), vocabulaire caractéristique de ce « crucial misunderstanding " relevé par Dalton.

Au-delà du présent ouvrage et de son auteur, peut-on utiliser ce terme autrement que par facilité de langage ? Il a été consigné pour la première fois par Xénophon, "économiste " par ailleurs, selon qui Socrate qualifiait de ploutocratie le gouvernement où les dirigeants sont désignés d'après leur richesse (Mémorables, 4, 6, 12), mais le mot manque dans le vocabulaire de Platon (pour qui la richesse, associée à la mollesse, sert uniquement de repoussoir à la tempérance) comme dans celui d'Aristote, de Polybe et des autres auteurs politiques grecs. Malgré son admiration pour le " génial » Pierre Leroux, qui publia en 1843 De la Ploutocratie, ou gouverne- ment des riches, Marx ne semble avoir utilisé le terme que dans un article consacré aux " conséquences futures de la domination britannique en Inde » (1853) où il distinguait les rôles respectifs de "l'aristocratie ", de la " ploutocratie " et de la " millocratie " (des propriétaires de fabriques ou « mills ») anglaises dans cette exploitation coloniale. Dans les pages de L'origine de la famille qu'il a consacrées à Athènes et à la Grèce antique et où le mot grec semblait s'imposer, Engels a préféré parler de Geldherrschaft. Renan a donné sa définition de la ploutocratie dans L'Avenir de la science (terminé en 1849 mais publié seulement en 1890) et la propagande antisémite s'en est probablement inspirée durant l'Affaire Dreyfus, mais c'est surtout l'audience des Systèmes socialistes (1902) de Vilfredo Pareto qui a assuré le succès du terme aussi bien dans le vocabulaire politique ("démocratie-ploutocratie ", refrain de La Libre Parole et de L'Action française) qu'auprès de sociologues comme Max Weber. Définir ce que le mot pourrait recouvrir dans les sociétés occidentales modernes étant déjà fort épineux, son application à une société " non intégrée aux échanges marchands » (Dalton, 1965 : 58) n'ira probablement pas non plus sans controverses.

Entre autres questions posées par le livre de J. Liep, il s'agirait aussi de savoir quelle est la visée principale des "ploutocrates » ou, comme écrivait Mauss, des détenteurs de "talismans » : « commander aux autres " du fait de leur "prestige ", ou s'enrichir soit pour étendre leur influence, soit pour thésauriser ? Du fait de la complexité des relations interpersonnelles et de « l'ambiguïté générale entourant les prêts, les gages et le crédit à Rossel » (Liep, p. 309), il semble difficile de le déterminer, sauf à plaquer des interprétations occidentales à la façon d'Armstrong. La notion de thésaurisation, qui pourrait apparemment s'appliquer à nombre de sociétés mélanésiennes et à celle-ci en particulier, a certes occupé Marx (1965 [1867] : notamment 672-676), mais comme l'a remarqué André Orléan (1991), selon une logique de "l'arbitraire individuel ", qui « relève essentiellement de la psychologie, et plus spécifiquement de la psychologie de l'avarice ", et faut-il ajouter, de celle du fétichisme. « Le thésaurisateur, écrit Marx, sacrifie à ce fétiche toute sa chair ». Il remplit néanmoins à son insu un rôle social essentiel, puisque « la partie d'or ou d'argent qui réellement circule ne doit former qu'une partie du métal précieux existant dans un pays. C'est par la forme trésor de l'argent que cette condition se trouve remplie. » Pour A. Orléan (1992: 124-125), "dans la thésaurisation, c'est bien la monnaie en tant que pouvoir qui est recherchée ", non plus comme moyen mais comme fin, introduisant une rupture dans " les sociétés holistes ", du fait du "regard critique " qu'elle introduit "vis-à-vis des représentations qui gouvernent les autres échangistes ». Mais 
Rossel a-t-elle réellement des "monnaies " et des thésaurisateurs auxquels pourraient s'appliquer ces remarques ? Le débat n'est pas clos. On doit admirer J. Liep de l'avoir si vigoureusement et si brillamment relancé.

\section{BIBLIOGRAPHIE}

Armstrong Wallace Edwin, 1978 [1928]. Rossel Island. An Ethnological Study. New York, AMS Press Reprints.

Dalton George, 1965. Primitive Money, American Anthropologist, New Series 67-1, pp. 4465.

De Coppet Daniel, 1970. 1, 4, 8, 9, 7. La monnaie : présence des morts et mesure du temps, L'Homme 10-1, pp. 17-39.

Derlon Brigitte, 2002. L'intestinal et le matriciel. Aux origines mythiques d'une " monnaie " mélanésienne (Nouvelle-Irlande, plateau Lelet), L'Homme 162, pp. 157-180.

EInzig Paul, 1949. Primitive Money in its ethnological, historical and economic aspects, London, Eyre and Spottiswoode (notamment chap. The intricate currency of Rossel Island, pp. 72-75).

Epstein E. L., 1979. Tambu: the Shell-Money of the Tolai, in R. H. Hook (ed.), Fantasy and Symbol. Studies in Anthropological Interpretation, New York, Academic Press, pp. 149-202.

Goldberg Dror, 2005. Famous Myths of 'Fiat Money', Journal of Money, Credit and Banking37 (5), Blackwell Publishing pp. 957967.

Küchler Susanne, 2002. Malangan. Art, Memory and Sacrifice, Oxford-New York, Berg.
Marx Karl, 1994 [1853]. Les conséquences futures de la domination britannique en Inde, in Chroniques anglaises, Euvres t. IV, Paris, Gallimard, Bibliothèque de la Pléiade, pp. 730-736.

-, 1965 [1867]. Le Capital, traduction par Joseph Roy, revue par Maximilien Rubel, in Euvres t. I, Paris, Gallimard, Bibliothèque de la Pléiade, pp. 535-1406.

Mauss Marcel, 1969 [1914]. Les origines de la notion de monnaie, in Euvres t. 2, Paris, Éditions de Minuit, pp. 106-112.

Monnerie Denis, 2002. Monnaies de Mono-Alu. Valeurs, discontinuités et continuités dans les objets et les relations sociales, L'Homme 162, pp. 80-105.

OrlÉAN André, 1991. L'origine de la monnaie (I), Revue du MAUSS 14, pp. 126-152.

—, 1992. L'origine de la monnaie (II). La monnaie dans les sociétés holistes, Revue du MAUSS 15-16, pp. 111-125.

PANOFF Michel, 1980. Objets précieux et moyens de paiement chez les Maenge de Nouvelle-Bretagne, L'Homme xx (2), pp. 5-37.

Robisns Joel, 2009. Equality, Inequality and Change, Suomen Antropologi 34 (4), pp. 7180.

Roheim Geza, 1978 [1923]. L'argent sacré en Mélanésie [Heiliges Geld in Melanesien], in Ernest Bornemann, Psychanalyse de l'argent. Une recherche critique sur les théories psychanalytiques de l'argent, Paris, Presses Universitaires de France, pp. 215-231.

WIEner Annette, 1992. Inalienable Possessions. The Paradox of keeping-while-giving, Berkeley, University of California Press. 
